\title{
Diferencia de tensión arterial en ambos brazos como medio para identificar pacientes con riesgo cardiovascular
}

\author{
Pedro García Martínez \\ perich97@gmail.com \\ ESTHER FERNÁNDEZ ARROYO \\ estherferarroyo@hotmail.com \\ ELADIO JOAQUín COLLADO BoIRA \\ colladoe@uji.es
}

\section{Resumen}

Introducción: Una diferencia de tensión arterial sistólica entre ambos brazos de $10 \mathrm{mmHg}$ o mayor se ha asociado a enfermedad vascular periférica, enfermedad cerebrovascular y aumento de mortalidad. El artículo estudia la diferencia de tensión arterial en ambos brazos de usuarios de una consulta de atención primaria. Secundariamente, busca asociar variables de género, edad e hipertensión para identificar perfiles de riesgo y su inclusión en estudios posteriores. Metodología: Estudio descriptivo transversal del total de pacientes atendidos en una consulta enfermera de atención primaria durante un mes. Se realiza toma de tensión arterial en ambos brazos, según protocolo. Se registran datos de género, edad, tensión arterial y diferencias interbraquiales de tensión arterial. Se realiza estudio estadístico descriptivo e inferencial mediante SPSS 20.0. Resultados: Población de 113 pacientes con cifras de tensión arterial sistólica media de 143,5 +/- 21,5 mm y diastólica media de $82,7+/-13,4 \mathrm{mmHg}$. Se observa diferencia superior a $10 \mathrm{mmHg}$ en tensión arterial sistólica interbraquial en $15,93 \%$ de la muestra. Se halla tendencia a la significación entre diferencia $>10 \mathrm{mmHg}$ de tensión con la edad, siendo significativa con diferencias $>15 \mathrm{mmHg}$. El género e hipertensión no muestran diferencias significativas en relación con la diferencia de tensión arterial interbraquial. Conclusiones: Los datos obtenidos de pacientes con diferencias de tensión arterial interbraquial son similares a otros estudios. Solo se identifica como factor de riesgo de los estudiados la edad, por lo que sería susceptible de realizar esta valoración a toda la población. Se propone protocolo de actuación en caso de identificación de pacientes con diferencias de tensión arterial interbraquial mayor de $10 \mathrm{mmHg}$.

Palabras clave: enfermería, atención primaria, tensión arterial, riesgo cardiovascular. 


\section{Abstract}

Introduction: A difference in systolic blood pressure in both arms of $10 \mathrm{mmHg}$ or greater has been associated with peripheral vascular disease, cerebrovascular disease and increased mortality. The article studies the difference in blood pressure in both arms of users of a primary care office. Secondarily, it looks for associated variables of gender, age and hypertension to identify risk profiles and their inclusion in future studies. Methodology: Descriptive transversal study of all the patients seen in a nurse-primary care office for a month. In both arms blood pressure is taken and performed according to protocol. It is registered data of gender, age, blood pressure and inter-arms blood pressure differences. The descriptive and inferential statistical study is done with SPSS 20.0. Results: Population of 113 patients with systolic blood pressure of $143.5 /-21.5 \mathrm{mmHg}$ and diastolic of $82.7 /-13.4 \mathrm{mmHg}$. It is observed a difference of more than $10 \mathrm{mmHg}$ in inter-arms systolic blood pressure at $15.93 \%$ of the study group. There is trend to significant difference between pressure higher than $10 \mathrm{mmHg}$ with age, being significant with differences higher than $15 \mathrm{mmHg}$. Gender and hypertension do not show significant differences in relation to the difference in inter-arms blood pressure. Conclusions: The data obtained from patients with inter-arms differences blood pressure are similar to other studies. Only age, is identified as a risk factor for the studied so it would be susceptible to conduct this assessment to the entire population. It is proposed protocol for action in case of identification of patients with inter-arms blood pressure differences greater than $10 \mathrm{mmHg}$.

Keywords: Nursing, primary atencion, blood pressure, cardiovascular risk.

\section{Introducción}

Una diferencia de tensión arterial sistólica entre ambos brazos de $10 \mathrm{mmHg}$ o mayor se ha asociado a enfermedad vascular periférica, enfermedad cerebrovascular preexistente y un aumento de la mortalidad por distintas patologías (Clark, 2012). Así se podría indicar que una diferencia igual o superior a $10 \mathrm{mmHg}$ ayudaría a identificar a pacientes que precisen una evaluación médica y una diferencia mayor de $15 \mathrm{mmHg}$ podría ser indicador de enfermedad cardiovascular y aumento de mortalidad en los pacientes (Clark, 2012).

La guía europea de manejo de la tensión arterial del 2007 señala que se debe «...determinar la presión arterial en ambos brazos en la primera visita para detectar posibles diferencias debidas a una enfermedad vascular periférica. En ese caso, hay que tomar como referencia el valor mayor...» (Mancia, 2007). Pero, como indica el Dr. Armario de la Sociedad Española de Hipertensión, ésta es una recomendación que no se sigue habitualmente en la práctica clínica (Armario, 2012).

Ya que es el equipo de enfermería uno de los encargados de la toma de tensión arterial en el centro de salud, incluida como actividad de diversos programas de salud dirigidos al paciente adulto y anciano, este artículo se propone estudiar las diferencias existentes en las cifras de tensión arterial tomadas en ambos brazos de los usuarios de la consulta de enfermería. De modo secundario, este estudio buscará los factores de riesgo asociados a diferencias de tensión arterial entre las variables de género, edad e hipertensión para poder encontrar criterios de inclusión para la realización de estudios posteriores. 


\section{Método}

Estudio descriptivo transversal de un cupo de pacientes del Departamento Diez de la Comunidad Valenciana, adscritos al centro de salud de Fuente de San Luis en el área urbana de la ciudad de Valencia, y cuya asistencia de salud corresponde a una sola enfermera. El total de la población del cupo es de 1363 pacientes y según los estudios publicados, se espera una población con diferencias de tensión arterial superiores a $15 \mathrm{mmHg}$ de un $7 \%$, por lo que el tamaño de muestra requerido para un nivel de confianza del $95 \%$ y un margen de error del $5 \%$ será de 94 usuarios. Para alcanzar esta población se decide incluir en el estudio a todos los pacientes que acuden a la consulta enfermera durante dos meses (junio y julio), ya sea a través de citas programadas, a demanda o de urgencia.

El protocolo para la toma de tensión se realiza en el interior de la consulta, con el paciente en sedestación y tras comprobar que haya pasado un mínimo de diez minutos de espera antes de entrar a la consulta y dos minutos desde la entrada del paciente. Para las características del manguito, aparato, control del mismo y metodología en la toma tensión arterial se siguen las recomendaciones de la Sociedad Europea de Hipertensión (Mancia, 2007). Son motivos de exclusión del estudio un tiempo de espera inferior a diez minutos, causas que provoquen agitación del paciente, los errores del tensiómetro que supongan la necesidad de repetir la toma en alguno de los dos brazos, la negativa del paciente a participar en el estudio o la derivación médica del paciente por estado grave del mismo.

El tensiómetro automático utilizado en todas las mediciones es del modelo OMRON M6 Comfort, y ha sido controlado el estado de calibración del mismo tres semanas antes de comenzar con el estudio. La técnica es realizada por la enfermera y el residente de enfermería familiar y comunitaria que han sido adiestrados en el protocolo de toma de tensión.

Las variables a estudio serán la edad, el género y la medida de tensión arterial sistólica y diastólica. Obtenidos estos datos se derivarán de ellas el estudio de las medias de tensión arterial sistólica y diastólica, y la diferencia en $\mathrm{mmHg}$ de las medidas en ambos brazo como variables cuantitativas. Por otro lado, se considerarán «cifras de hipertensión arterial sistólica» aquellas que superen $140 \mathrm{mmHg}$; y «de hipertensión arterial diastólica», las que sean superior a $90 \mathrm{mmHg}$ (Mancia, 2007). Por último, se realizará una separación entre los pacientes que tengan diferencias de tensión arterial sístólica entre ambos brazos mayor o igual a $10 \mathrm{mmHg}$ y aquellos que no presenten estas cifras; así como la separación con cifras iguales o superiores a $15 \mathrm{mmHg}$.

El estudio estadístico de las variables anteriores se realizará mediante el paquete informático SPSS 20.0. En el estudio estadístico descriptivo, las variables cualitativas se presentarán mediante el número absoluto y porcentaje, y las variables cuantitativas se presentarán mediante la media y desviación estándar de las mismas. Para el análisis inferencial de los datos se realizará mediante test ANOVA para el cruce de variables cuantitavas y cualitavas, y en las variables cualitativas mediante el test de chi cuadrado. Se considerará significativos los estudios que presenten un nivel de significación inferior a 0,05.

\section{Resultados}

De los 138 usuarios potenciales que acuden a consulta de enfermería durante el periodo de estudio, $25(18,1 \%)$ de ellos son excluidos: 17 usuarios por no haber permanecido el tiempo mínimo de 10 minutos en espera antes de entrar a la consulta, 1 usuario se excluyó por presentar una lesión en un brazo que impedía la toma de tensión arterial, 5 por fallos en 
el tensiómetro y 2 por interrupciones de carácter urgente durante la visita. Con un total de 113 usuarios se supera ampliamente los 94 pacientes requeridos para la muestra.

De los 113 usuarios que participaron definitivamente en el estudio fueron 41 (36,3\%) hombres y $72(63,7 \%)$ mujeres con una edad media global de 72,8 +/- 11,4 años. Las cifras de tensión arterial sistólica de la población fueron de 143,5 +/- 21,5 $\mathrm{mmHg}$ en el brazo izquierdo y $141,7+/-21,4 \mathrm{mmHg}$ en el derecho. Las cifras de tensión arterial diastólica fue de 82,7 +/13,4 y $82,3+/-13,9 \mathrm{mmHg}$ en los brazos izquierdo y derecho, respectivamente. Las diferencias halladas entre ambos brazos fueron de 1,83+/-10,2 mmHg en la tensión sistólica y 0,36+/-8,2 $\mathrm{mmHg}$ en la diastólica, como se muestra en la tabla 1.

Tabla 1

Variables cuantitativas en las que se muestran media, desviación estándar, la cifra mínima y máxima

\begin{tabular}{lcccc}
\hline & Media & d. t. & Mínimo & Máximo \\
\hline Edad & 72,82 & 11,43 & 39 & 92 \\
TAS izq. & 143,53 & 21,56 & 105 & 215 \\
TAD izq. & 82,71 & 13,46 & 55 & 130 \\
TAS der. & 141,70 & 21,38 & 101 & 206 \\
TAD der. & 82,34 & 13,95 & 49 & 150 \\
TAS media & 142,61 & 20,85 & 106 & 210 \\
TAD media & 82,53 & 13,07 & 52 & 140 \\
Dif. TAS & 1,83 & 10,25 & -26 & 44 \\
Dif. TAD & 0,36 & 8,25 & -20 & 34 \\
\hline
\end{tabular}

Nota. TAS: tensión arterial sistólica; TAD: tensión arterial diastólica; izq.: izquierda; der.: derecha. Media: media aritmética de la tensión calculada en ambos brazos. Dif.: diferencia entre las cifras de ambos brazos.

El porcentaje de hipertensión sistólica es de $52,2 \%$ en brazo izquierdo y de $46.9 \%$ en el derecho; en la diastólica se encuentra un $24,8 \%$ y $19,5 \%$ de hipertensos en los brazos izquierdo y derecho respectivamente. La diferencia de tensión arterial entre ambos brazos superior a $10 \mathrm{mmHg}$ aparece en un 15,9\% $(n=18)$ en la tensión sistólica y en un $7,1 \%(n=8)$ en diastólica. Asimismo, encontramos diferencias superiores a $15 \mathrm{mmHg}$ en sistólica en un $7,1 \%$ $(n=8)$ y en diastólica a un $4,4 \%(n=5)$, como se muestra en la tabla 2.

Tabla 2

Variables cualitativas en las que se muestran N: número absoluto de hombres en género y los pacientes que cumplen los requisitos de hipertensión o de diferencias de tensión arterial iguales o superiores a la cifra indicada

\begin{tabular}{lll}
\hline & N & $\%$ \\
\hline Género (hombre) & 41 & 36,28 \\
HTAS izq. & 59 & 52,21 \\
HTAD izq. & 28 & 24,78
\end{tabular}




\begin{tabular}{lll}
\hline & $N$ & $\%$ \\
\hline HTAS der. & 53 & 46,90 \\
HTAD der. & 22 & 19,47 \\
HTAS media & 58 & 51,33 \\
HTAD media & 27 & 23,89 \\
Dif. TAS $>10 \mathrm{mmHg}$ & 18 & 15,93 \\
Dif. TAD $>10 \mathrm{mmHg}$ & 8 & 7,08 \\
Dif. TAS $>15 \mathrm{mmHg}$ & 8 & 7,08 \\
Dif. TAD $>15 \mathrm{mmHg}$ & 5 & 4.42 \\
\hline
\end{tabular}

Nota. HTAS: hipertensión arterial sistólica superior a $140 \mathrm{mmHg}$; HTAD: hipertensión arterial diastólica superior a $90 \mathrm{mmHg}$; izq.: izquierda; der.: derecha. Dif.: diferencia entre las cifras de ambos brazos.

La tabla 3 muestra el estudio comparativo mediante ji cuadrado del género con las distintas variables y solo muestra tendencia a la significación en el caso de diferencias de tensión arterial superior a $15 \mathrm{mmHg}$ : siendo mayor la presencia de esta característica en hombres que en mujeres.

Tabla 3

Estudio descriptivo analítico mediante la prueba de ji cuadrado

\begin{tabular}{lrrrrr}
\hline & \multicolumn{2}{c}{ Hombres } & \multicolumn{2}{c}{ Mujeres } & $p$ \\
\hline HTAS izq. & $\mathrm{N}$ & $\%$ & $\mathrm{~N}$ & $\%$ & \\
HTAD izq. & 21 & 51,22 & 38 & 52,78 & 0,873 \\
HTAS der. & 8 & 19,50 & 20 & 27,80 & 0,328 \\
HTAD der. & 21 & 51,22 & 32 & 44,40 & 0,488 \\
HTAS media & 7 & 17,02 & 15 & 20,80 & 0,627 \\
HTAD media & 21 & 51,22 & 37 & 51,39 & 0,986 \\
Dif. TAS $>10 \mathrm{mmHg}$ & 7 & 17,02 & 20 & 27,80 & 0,199 \\
Dif. TAD $>10 \mathrm{mmHg}$ & 5 & 12,20 & 13 & 18,06 & 0,413 \\
Dif. TAS $>15 \mathrm{mmHg}$ & 4 & 9,76 & 4 & 5,56 & 0,458 \\
Dif. TAD $>15 \mathrm{mmHg}$ & 2 & 4,88 & 6 & 8,33 & 0,709 \\
\hline
\end{tabular}

Nota. Se considera significativo cuando el nivel de significación es inferior a 0,05 y tendente a la significación con cifras inferiores a 0,1 . N: número absoluto de hombres en género y los pacientes que cumplen los requisitos de hipertensión o de diferencias de tensión arterial iguales o superiores a la cifra indicada. P: nivel de signifiación. HTAS: hipertensión arterial sistólica superior a $140 \mathrm{mmHg}$; HTAD: hipertensión arterial diastólica superior a $90 \mathrm{mmHg}$; izq: izquierda; der: derecha. Dif: diferencia entre las cifras de ambos brazos. 
Las tablas 4 y 5 muestran el estudio de los pacientes que cumplen el criterio de diferencias de tensión arterial sistólica superior a 10 y $15 \mathrm{mmHg}$, respectivamente, entre ambos brazos comparado con pacientes con hipertensión arterial sistólica en ambos brazos y con los que presentan diferencias de tensión superiores a 15 o $10 \mathrm{mmHg}$.

Tabla 4

Estudio descriptivo analítico mediante la prueba de ji cuadrado

\begin{tabular}{lrcccr}
\hline & \multicolumn{2}{c}{ Dif. TAS $>10 \mathrm{mmHg}$} & \multicolumn{2}{c}{ Dif. TAS $<10 \mathrm{mmHg}$} & $p$ \\
\hline HTAS izq. & $\mathrm{N}$ & $\%$ & $\mathrm{~N}$ & $\%$ & \\
HTAS der. & 12 & 66,7 & 47 & 49,5 & 0,207 \\
Dif. TAS $>15 \mathrm{mmHg}$ & 8 & 44,4 & 45 & 47,4 & 1,000 \\
\hline
\end{tabular}

Nota. Se considera significativo cuando el nivel de significación es inferior a 0,05 y tendente a la significación con cifras inferiores a 0,1 . N: número absoluto de pacientes que cumplen los criterios de cada fila. P: nivel de signifiación. HTAS: hipertensión arterial sistólica superior a $140 \mathrm{mmHg}$; izq.: izquierda; der.: derecha. Dif.: diferencia entre las cifras de ambos brazos.

Tabla 5

Estudio descriptivo analítico mediante la prueba de ji cuadrado

\begin{tabular}{lccccr}
\hline & \multicolumn{2}{c}{ Dif. TAS $>15 \mathrm{mmHg}$} & \multicolumn{2}{c}{ Dif. TAS $<15 \mathrm{mmHg}$} & $p$ \\
\hline HTAS izq. & $\mathrm{N}$ & $\%$ & $\mathrm{~N}$ & $\%$ & \\
HTAS der. & 7 & 87,5 & 52 & 49,5 & 0,063 \\
Dif. TAS $>10 \mathrm{mmHg}$ & 4 & 50,0 & 49 & 46,7 & 1,000 \\
\hline
\end{tabular}

Nota. Se considera significativo cuando el nivel de significación es inferior a 0,05 y tendente a la significación con cifras inferiores a 0,1 . N: número absoluto de pacientes que cumplen los criterios de cada fila. P: nivel de signifiación. HTAS: hipertensión arterial sistólica superior a $140 \mathrm{mmHg}$; izq.: izquierda; der.: derecha. Dif.: diferencia entre las cifras de ambos brazos.

Por otro lado, la relación de la tensión arterial con la edad muestra ser significativo en las cifras de hipertensión arterial sistólica en brazo izquierdo (75.6+/-9.9 años en los hipertensos por un $69,8+/-12,1$ años en los no hipertensos) y en brazo derecho $(75,1+/-10,2$ años versus $70,8+/-12,1$ años). Asimismo, la diferencia mayor de $15 \mathrm{mmHg}$ en la tensión arterial sistólica en ambos brazos también es significativa siendo la edad media de los que presentan esta diferencia de $81,2+/-4,9$ años por $72,2+/-11,5$ los que no presentan diferencias tan importantes (tabla 6). 
Tabla 6

Estudio descriptivo analítico mediante la prueba ANOVA de un factor para la variable edad

\begin{tabular}{lccccc}
\hline & \multicolumn{2}{c}{ No } & Sí & $p$ \\
\hline & Media & d. t. & Media & d. t. & \\
\hline HTAS izq. & 69,78 & 12,19 & 75,61 & 9,99 & 0,006 \\
HTAD izq. & 73,40 & 11,87 & 71,07 & 9,97 & 0,352 \\
HTAS der. & 70,80 & 12,14 & 75,11 & 10,20 & 0,045 \\
HTAD der. & 73,75 & 11,75 & 69,00 & 9,25 & 0,080 \\
Dif. TAS >10 mmHg & 72,13 & 11,34 & 76,50 & 11,53 & 0,137 \\
Dif. TAD >10 mmHg & 72,49 & 11,64 & 77,25 & 7,25 & 0,258 \\
Dif. TAS >15 mmHg & 72,18 & 11,54 & 81,25 & 4,95 & 0,030 \\
Dif. TAD >15 mmHg & 72,57 & 11,50 & 78,20 & 9,09 & 0,284 \\
\hline
\end{tabular}

Nota. Se considera significativo cuando el nivel de significación es inferior a 0,05 y tendente a la significación con cifras inferiores a 0 ,1. Media: edad media de los que cumplen el criterio. HTAS: hipertensión arterial sistólica superior a $140 \mathrm{mmHg}$; HTAD: hipertensión arterial diastólica superior a $90 \mathrm{mmHg}$; izq.: izquierda; der.: derecha. Dif.: diferencia entre las cifras de ambos brazos.

\section{Discusión y conclusiones}

Si tomamos las directrices de la Guía Europea de Hipertensión (Mancia, 2007) y consideramos como pacientes hipertensos aquellos que presentan cifras superiores a $140 \mathrm{mmHg}$ de tensión arterial sistólica y $90 \mathrm{mmHg}$ de tensión arterial diastólica tomadas en el brazo de cifras más elevadas, encontramos que nuestra población presenta cifras de hipertensión sistólica de un $52,21 \%$ en el brazo izquierdo y de hipertensión diastólica en un $24,78 \%$ en el mismo brazo. Estas cifras serían superiores a las demostradas en el estudio ENRICA del año 2010, que muestra una prevalencia de hipertensión del $33 \%$ en la población general; pero al encontrar una edad media superior a 70 años en nuestra muestra, estas cifras estarían por debajo de lo esperado en mayores de 60 años, con prevalencia de más del $60 \%$ de hipertensos (Kearney, 2005; Banegas, 2002).

En las diferencias de tensión arterial sistólica, objetivo de este estudio, observamos que el $15,9 \%$ de pacientes presentan cifras superiores a $10 \mathrm{mmHg}$ de diferencia entre ambos brazos, lo que supone un porcentaje de población superior al compararlo con el $14 \%$ mostrado por el metanálisis de Verbek (2011), lo que podría suponer un aumento de riesgo de mortalidad del $24 \%$ o mayor, según estudios internacionales (Agarwal, 2008). Pero si atendemos a las diferencias superiores a $15 \mathrm{mmHg}$, la población estudiada presenta cifras similares a las mostradas por otros estudios (Clark, 2012) con cifras del 7 \% de población afecta por esta diferencia de tensión interbraquial.

Si bien la sensibilidad de asociación entre diferencias superiores a $15 \mathrm{mmHg}$ entre los brazos y la patología vascular periférica no es muy alta (15\%), su especificidad sí que los es (96 \%) (Clark, 2012). Así que aunque no sea una buena prueba para el despistaje de la patología vascular periférica, sí que sería un buen indicador para la realización de interconsulta con el médico de familia y realizar la medición del índice tobillo brazo, más sensible para dicho despistaje (Mancia, 2007).

No se han encontrado relaciones importantes entre las diferencias de tensión arterial sistólica entre los brazos y las variables de hipertensión sistólica, diastólica o media, por lo que 
no se podría excluir a ningún paciente de dicha valoración. En cuanto al género se ha mostrado una tendencia a la significación en el caso de los hombres, pero debido a las limitaciones de la muestra sería aconsejable aumentar el tamaño de la población a estudio para comprobar esta relación. Por otro lado, sí que se observa relación con la edad, ya que es más habitual encontrar diferencias altas de tensión arterial sistólica en los pacientes de mayor edad. Esta situación es esperable al ser la edad un importante factor de riesgo en la patología cardiovascular como presenta la escala SCORE (De Backer, 2003).

En conclusión, podríamos decir que el estudio de la tensión arterial en ambos brazos aporta importante información sobre los riesgos cardiovasculares del paciente y, aunque no pueda usarse como screening de patología cardiovascular, puede ser un factor que inste a enfermería a realizar una valoración del índice tobillo brazo y derivación al médico de familia.

Con nuestros datos no se podría excluir a nadie del estudio de la tensión en ambos brazos, ya que no existen factores de riesgo claros relacionados con una toma aislada de tensión arterial, y todos los usuarios podrían beneficiarse de esta técnica. Una limitación de este estudio es no haber incluido las patologías y tratamientos farmacológicos de los pacientes que podrían ser factor de riesgo para la diferencia de tensión arterial interbraquial, pero el objetivo inicial era el estudio de la población basados en la toma de constantes por parte de enfermería.

Por último, destacar que este estudio presenta las limitaciones propias de una población pequeña y limitada a un cupo exclusivo, por lo que sería difícil la extrapolación de los datos a otras poblaciones y, por este motivo, sería interesante continuar esta valoración con poblaciones más amplias. Con estudios más amplios se podría intentar recuperar la toma de tensión en ambos brazos e iniciar un protocolo para la derivación al médico de familia y el estudio del índice tobillo brazo en aquellos pacientes que presenten diferencias superiores a $10 \mathrm{mmHg}$, independientemente de sus patologías crónicas diagnosticadas, ya que esta actuación es incruenta, barata y no requiere especial formación para el equipo de enfermería.

\section{Referencias bibliográficas}

Agarwal, R., Bunaye, Z., y Bekele, D. M. (2008). Hypertension detection and control. Prognostic significance of between-arm blood pressure differences. Hypertension, 51, 657-662.

Armario, P. (2012). Asociación de la diferencia de presión arterial sistólica entre ambos brazos con la enfermedad vascular y la mortalidad. Recuperado de http://www.seh-lelha.org/ articulosr.aspx el 16 de julio de 2012.

Banegas, J. R., Rodríguez Artalejo, F. (2002). El problema de la hipertensión arterial en España. Revista Clínica Española, 202, 12-15.

Banegas, J. R. y Artalejo, R. (2011). Estudio ENRICA. Presentado en el XVI Congreso Nacional de la SEH, Barcelona.

Clark, C. E., Taylor, R. S., Shore, A. C., Ukoummunne, O. C., Campbell, J. L. (2012). Association of a difference in systolic blood pressure between arms with vascular disease and mortality: a systematic review and meta-analysis. The Lancet, 379, 905-914.

Conroy, R. M., Pyörälä, K., Fitzgerald, A. P., Sans, S., Menotti, A., De Backer, G., ... y scoRE project group. (2003). Estimation of ten-year risk of fatal cardiovascular disease in Europe: the SCORE project. European Heart Journal, 24, 987-1003.

Kearney, P. M., Whelton, M., Reynolds, K., Muntner, P., Whelton, P. K., y He, J. (2005). Global burden of hypertension: analysis of worldwide data. The Lancet, 365, 217-23.

Mancia, G., de Backer, G., Dominiczak, A., Cifkova, R., Fagard, R., Germano, G., ... Williams, B. (2007). 2007 Guidelines for the Management of Arterial Hypertension: The Task Force for 
the Management of Arterial Hypertension of the European Society of Hypertension (ESH) and of the European Society of Cardiology (ESC). Journal of Hypertension, 25, 1105-87.

Verberk, W. J., Kessels, A. G., y Thien, T. (2011). Blood pressure measurement method and inter-arm differences: a meta-analysis. American Journal of Hypertension, 24, 12011208. 